\title{
BOOLEAN METHODS FOR DOUBLE INTEGRATION
}

\author{
FRANZ-J. DELVOS
}

\begin{abstract}
This paper is concerned with numerical integration of continuous functions over the unit square $U^{2}$. The concept of the $r$ th-order blending rectangle rule is introduced by carrying over the idea from Boolean interpolation. Error bounds are developed, and it is shown that $r$ th-order blending rectangle rules are comparable with number-theoretic cubature rules. Moreover, $r$ thorder blending midpoint rules are defined and compared with the $r$ th-order blending rectangle rules.
\end{abstract}

\section{Bivariate RectANGle RUles}

The problem we consider is the numerical evaluation of integrals of the form

$$
\mathfrak{I}(f)=\int_{0}^{1} \int_{0}^{1} f(x, y) d x d y,
$$

where $f$ is a continuous function on the unit square $U^{2}=[0,1]^{2}$. Moreover, we assume that $f$ satisfies the periodicity conditions

$$
f(x, 0)=f(x, 1), \quad f(0, y)=f(1, y) \quad(0 \leq x, y \leq 1) .
$$

The inner product of $f, g \in L^{2}\left(U^{2}\right)$ is

$$
(f, g)=\int_{0}^{1} \int_{0}^{1} f(x, y) \overline{g(x, y)} d x d y .
$$

We introduce the notations

$$
\begin{aligned}
e_{k}(x) & =\exp (i 2 \pi k x) \quad(k \in \mathbb{Z}), \\
e_{k, l}(x, y) & =e_{k}(x) \cdot e_{l}(y) \quad(k, l \in \mathbb{Z}),
\end{aligned}
$$

where $x, y \in U$. The functions $e_{k, l}(k, l \in \mathbb{Z})$ form an orthonormal basis of the Hilbert space $L^{2}\left(U^{2}\right)$. We denote by $A\left(U^{2}\right)$ the Wiener algebra of those functions $f \in L^{2}\left(U^{2}\right)$ with the property that the Fourier series of $f$ is absolutely convergent:

$$
\sum_{k=-\infty}^{\infty} \sum_{l=-\infty}^{\infty}\left|\left(f, e_{k, l}\right)\right|<\infty .
$$

Received April 3, 1989; revised November 30, 1989.

1980 Mathematics Subject Classification (1985 Revision). Primary 65D30, 65D32.

Key words and phrases. Multiple integration, blending methods. 
Let $\mathscr{C}\left(U^{2}\right)$ denote the subspace of those functions $f \in L^{2}\left(U^{2}\right)$ which are continuous on $U^{2}$. Moreover, $\mathscr{C}_{0}\left(U^{2}\right)$ denotes the subspace of those functions $f \in \mathscr{C}\left(U^{2}\right)$ which satisfy the periodicity conditions (1.2). It follows from relation (1.3) that

$$
A\left(U^{2}\right) \subseteq \mathscr{C}_{0}\left(U^{2}\right)
$$

and, for $f \in A\left(U^{2}\right)$,

$$
f(x, y)=\sum_{k=-\infty}^{\infty} \sum_{l=-\infty}^{\infty}\left(f, e_{k, l}\right) \cdot e_{k, l}(x, y) \quad(x, y \in U) .
$$

Let $m$ and $n$ be positive integers. The most obvious cubature formula is the bivariate rectangle rule:

$$
\mathfrak{I}_{m, n}(f)=\frac{1}{m \cdot n} \sum_{j=0}^{m-1} \sum_{k=0}^{n-1} f\left(\frac{j}{m}, \frac{k}{n}\right) .
$$

The bivariate rectangle rule is not an efficient cubature formula in view of the large number of function evaluations. On the other hand, $\mathfrak{I}_{m, n}(f)$ is a basic tool in constructing a more sophisticated cubature formula, the $r$ th-order blending rectangle rule. For this reason we will briefly derive a convenient remainder formula for $\mathfrak{I}_{m, n}(f)$.

Proposition 1. If $f \in A\left(U^{2}\right)$, then

$$
\mathfrak{I}_{m, n}(f)=\sum_{u=-\infty}^{\infty} \sum_{v=-\infty}^{\infty}\left(f, e_{u m, v n}\right) .
$$

Proof. In view of (1.4), we have

$$
\begin{aligned}
\mathfrak{I}_{m, n}(f) & =\frac{1}{m \cdot n} \sum_{j=0}^{m-1} \sum_{k=0}^{n-1} f\left(\frac{j}{m}, \frac{k}{n}\right) \\
& =\sum_{r=-\infty}^{\infty} \sum_{s=-\infty}^{\infty}\left(f, e_{r, s}\right) \frac{1}{m \cdot n} \sum_{j=0}^{m-1} e_{r}\left(\frac{j}{m}\right) \sum_{k=0}^{n-1} e_{s}\left(\frac{k}{n}\right) \\
& =\sum_{u=-\infty}^{\infty} \sum_{v=-\infty}^{\infty}\left(f, e_{u m, v n}\right) .
\end{aligned}
$$

It is useful to define the series

$$
\begin{gathered}
R_{m, \infty}(f)=\sum_{u \neq 0}\left(f, e_{u m, 0}\right), \quad R_{\infty, n}(f)=\sum_{v \neq 0}\left(f, e_{0, v n}\right), \\
R_{m, n}(f)=\sum_{u \neq 0} \sum_{v \neq 0}\left(f, e_{u m, v n}\right) .
\end{gathered}
$$

Proposition 2. If $f \in A\left(U^{2}\right)$, then the error in the bivariate rectangle rule is

$$
\mathfrak{I}_{m, n}(f)-\mathfrak{I}(f)=R_{m, \infty}(f)+R_{\infty, n}(f)+R_{m, n}(f) .
$$


Proof. It follows from relation (1.5) that

$$
\mathfrak{I}_{m, n}(f)=\left(f, e_{0,0}\right)+R_{m, \infty}(f)+R_{\infty, n}(f)+R_{m, n}(f) .
$$

Since $\mathfrak{I}(f)=\left(f, e_{0,0}\right)$, Proposition 2 is proved.

Following Korobov, we define, for each $a \geq 1$, the linear space

$$
E^{a}\left(U^{2}\right)=\left\{f \in L^{2}\left(U^{2}\right):\left(f, e_{m, n}\right)=\mathscr{O}\left((\bar{m} \cdot \bar{n})^{-a}\right)(m, n \rightarrow \infty)\right\},
$$

where $\bar{m}=\max \{1,|m|\} \quad(m \in \mathbb{Z})$. It is easily seen that

$$
E^{a}\left(U^{2}\right) \subseteq A\left(U^{2}\right) \quad(a>1) .
$$

We denote by $\mathscr{C}^{p, p}\left(U^{2}\right)$ the linear subspace of $\mathscr{C}\left(U^{2}\right)$ of those functions $f$ whose partial derivatives satisfy

$$
D^{k, l} f \in \mathscr{C}\left(U^{2}\right) \quad(0 \leq k, l \leq p) .
$$

Similarly, $\mathscr{C}_{0}^{p, p}\left(U^{2}\right)$ is the linear subspace of $\mathscr{C}_{0}\left(U^{2}\right)$ of functions $f$ with

$$
D^{k, l} f \in \mathscr{C}_{0}\left(U^{2}\right) \quad(0 \leq k, l \leq p) .
$$

It was shown in Baszenski and Delvos [1] that

$$
\mathscr{C}_{0}^{q-1, q-1}\left(U^{2}\right) \cap \mathscr{C}^{q+1, q+1}\left(U^{2}\right) \subseteq E^{q+1}\left(U^{2}\right) \quad(q \in \mathbb{N}) .
$$

Proposition 3. If $f \in E^{a}\left(U^{2}\right)$ with $a>1$, then the error in the bivariate rectangle rule satisfies

$$
\mathfrak{I}_{m, n}(f)-\mathfrak{I}(f)=\mathscr{O}\left(m^{-a}+n^{-a}\right) \quad(m, n \rightarrow \infty) .
$$

Proof. Since $f \in E^{a}\left(U^{2}\right)$, we have

$$
\begin{gathered}
R_{m, \infty}(f)=\mathscr{O}\left(m^{-a}\right), \quad R_{\infty, n}(f)=\mathscr{O}\left(n^{-a}\right), \\
R_{m, n}(f)=\mathscr{O}\left(m^{-a} \cdot n^{-a}\right) \quad(m, n \rightarrow \infty),
\end{gathered}
$$

from which (1.9) follows by virtue of Proposition 2.

Proposition 4. If $f \in \mathscr{C}_{0}^{q-1, q-1}\left(U^{2}\right) \cap \mathscr{C}^{q+1, q+1}\left(U^{2}\right)$ with $q \in \mathbb{N}$, then the error in the bivariate rectangle rule satisfies

$$
\mathfrak{I}_{m, n}(f)-\mathfrak{I}(f)=\mathscr{O}\left(m^{-q-1}+n^{-q-1}\right) \quad(m, n \rightarrow \infty) .
$$

Proof. Using (1.8), an application of Proposition 3 yields (1.11)

\section{2. $r$ TH-ORDER BLENDING RECTANGLE RULES}

We introduce the $r$ th-order sum of bivariate rectangle rules

$$
\mathbf{S}_{r}^{2}(f)=\sum_{m=1}^{r} \mathfrak{I}_{2^{m}, 2^{r+1-m}}(f) \quad\left(r \in \mathbb{Z}_{+}\right) .
$$

Then the rth-order blending rectangle rule $\mathfrak{I}_{r}^{2}(f)$ is

$$
\mathfrak{I}_{r}^{2}(f)=\mathbf{S}_{r}^{2}(f)-\mathbf{S}_{r-1}^{2}(f)
$$


where $r \in \mathbb{N}$ and $r>1$. The construction of the $r$ th-order blending rectangle rule resembles the explicit formula of the interpolation projector of $r$ th-order blending (Delvos and Posdorf [3] and Delvos [2]). The cubature points of $\mathfrak{I}_{r}^{2}(f)$ are mainly determined by the points occurring in $\mathbf{S}_{r}^{2}(f)$ :

$$
\bigcup_{m=1}^{r}\left\{\left(j \cdot 2^{-m}, k \cdot 2^{-r-1+m}\right): 0 \leq j<2^{m}, 0 \leq k<2^{r+1-m}\right\} .
$$

Their number is given by

$$
n_{r}=(r+1) \cdot 2^{r} .
$$

Next we will determine a remainder formula for the $r$ th-order blending rectangle rule.

Proposition 5. If $f \in A\left(U^{2}\right)$, then the error in the rth-order blending rectangle rule is

$$
\begin{aligned}
\mathfrak{I}_{r}^{2}(f)-\mathfrak{I}(f)= & R_{2^{r}, \infty}(f)+R_{\infty, 2^{r}}(f) \\
& +\sum_{m=1}^{r} R_{2^{m}, 2^{r+1-m}}(f)-\sum_{m=1}^{r-1} R_{2^{m}, 2^{r-m}}(f) .
\end{aligned}
$$

Proof. Using (1.6), we get

$$
\begin{aligned}
\mathfrak{I}_{r}^{2}(f)-\mathfrak{I}(f)= & \sum_{m=1}^{r}\left(\mathfrak{I}_{2^{m}, 2^{r+1-m}}(f)-\mathfrak{I}(f)\right)-\sum_{m=1}^{r-1}\left(\mathfrak{I}_{2^{m}, 2^{2-m}}(f)-\mathfrak{I}(f)\right) \\
= & \sum_{m=1}^{r}\left(R_{2^{m}, 2^{r+1-m}}(f)+R_{2^{m}, \infty}(f)+R_{\infty, 2^{m}}(f)\right) \\
& -\sum_{m=1}^{r-1}\left(R_{2^{m}, 2^{r-m}}(f)+R_{2^{m}, \infty}(f)+R_{\infty, 2^{m}}(f)\right) \\
= & R_{2^{r}, \infty}(f)+R_{\infty}, 2^{r}(f) \\
& +\sum_{m=1}^{r} R_{2^{m}, 2^{r+1-m}}(f)-\sum_{m=1}^{r-1} R_{2^{m}, 2^{r-m}}(f) . \quad \square
\end{aligned}
$$

Proposition 6. If $f \in E^{a}\left(U^{2}\right)$ with $a>1$, then the error in the rth-order blending rectangle rule is

$$
\mathfrak{I}_{r}^{2}(f)-\mathfrak{I}(f)=\mathscr{O}\left((r+1) \cdot\left(2^{r}\right)^{-a}\right) \quad(r \rightarrow \infty) .
$$

Proof. From (1.10) we have

$$
\begin{gathered}
R_{2^{r}, \infty}(f)=\mathscr{O}\left(\left(2^{r}\right)^{-a}\right), \quad R_{\infty, 2^{r}}(f)=\mathscr{O}\left(\left(2^{r}\right)^{-a}\right) \quad(r \rightarrow \infty), \\
R_{2^{m}, 2^{r+1-m}}(f)=\mathscr{O}\left(\left(2^{r+1}\right)^{-a}\right) \quad(1 \leq m \leq r, r \rightarrow \infty), \\
R_{2^{m}, 2^{r-m}}(f)=\mathscr{O}\left(\left(2^{r}\right)^{-a}\right) \quad(1 \leq m<r, r \rightarrow \infty) .
\end{gathered}
$$

Now (2.6) follows from the remainder formula (2.5). 
Remark 1. Recall that the number of cubature points of the $r$ th-order blending rectangle rule $\mathfrak{I}_{r}^{2}(f)$ is bounded by

$$
n_{r}=(r+1) 2^{r} \text {. }
$$

It is easily seen that the error relation (2.6) of the $r$ th-order blending rectangle rule obtains the form

$$
\mathfrak{I}_{r}^{2}(f)-\mathfrak{I}(f)=\mathscr{O}\left(\log \left(n_{r}\right)^{a+1} \cdot\left(n_{r}\right)^{-a}\right) \quad(r \rightarrow \infty),
$$

where $f \in E^{a}\left(U^{2}\right)$ with $a>1$. Thus, the $r$ th-order blending rectangle rule is comparable with the bivariate number-theoretic "good-lattice" rules (see Sloan [5]). The attractive feature of the $r$ th-order blending rectangle rule is its easy computation based on relations (2.1) and (2.2).

Proposition 7. If $f \in \mathscr{C}_{0}^{q-1, q-1}\left(U^{2}\right) \cap \mathscr{C}^{q+1, q+1}\left(U^{2}\right)$ with $q \in \mathbb{N}$, then the error in the rth-order blending rectangle rule satisfies

$$
\mathfrak{I}_{r}^{2}(f)-\mathfrak{I}(f)=\mathscr{O}\left((r+1) \cdot\left(2^{r}\right)^{-q-1}\right) \quad(r \rightarrow \infty) .
$$

Proof. Use of (1.8) and an application of Proposition 6 yields (2.7).

\section{BIVARIATE MIDPOINT RULES}

Let $m$ and $n$ be positive integers. A simple cubature formula closely related to the bivariate rectangle rule is the bivariate midpoint rule:

$$
\mathfrak{M}_{m, n}(f)=\frac{1}{m \cdot n} \sum_{j=0}^{m-1} \sum_{k=0}^{n-1} f\left(\frac{2 j+1}{2 m}, \frac{2 k+1}{2 n}\right) .
$$

Again, the bivariate midpoint rule is not an efficient cubature formula in view of the large number of function evaluations. However, $\mathfrak{M}_{m, n}(f)$ is a basic tool in constructing the more sophisticated cubature formula of the $r$ th-order blending midpoint rule. For this reason we will briefly derive a convenient remainder formula for $\mathfrak{M}_{m, n}(f)$.

Proposition 8. If $f \in A\left(U^{2}\right)$, then

$$
\mathfrak{M}_{m, n}(f)=\sum_{u=-\infty}^{\infty} \sum_{v=-\infty}^{\infty}\left(f, e_{u m, v n}\right) \cdot(-1)^{u+v} .
$$

Proof. By (1.4), we have

$$
\begin{aligned}
\mathfrak{M}_{m, n}(f) & =\frac{1}{m \cdot n} \sum_{j=0}^{m-1} \sum_{k=0}^{n-1} f\left(\frac{2 j+1}{2 m}, \frac{2 k+1}{2 n}\right) \\
& =\sum_{r=-\infty}^{\infty} \sum_{s=-\infty}^{\infty}\left(f, e_{r, s}\right) \frac{1}{m \cdot n} \sum_{j=0}^{m-1} e_{r}\left(\frac{2 j+1}{2 m}\right) \sum_{k=0}^{n-1} e_{s}\left(\frac{2 k+1}{2 n}\right) \\
& =\sum_{u=-\infty}^{\infty} \sum_{v=-\infty}^{\infty}\left(f, e_{u m, v n}\right) \cdot(-1)^{u+v} \cdot
\end{aligned}
$$


We define the series

$$
\begin{aligned}
Q_{m, \infty}(f) & =\sum_{u \neq 0}\left(f, e_{u m, 0}\right) \cdot(-1)^{u}, \\
Q_{\infty, n}(f) & =\sum_{v \neq 0}\left(f, e_{0, v n}\right) \cdot(-1)^{v}, \\
Q_{m, n}(f) & =\sum_{u \neq 0} \sum_{v \neq 0}\left(f, e_{u m, v n}\right) \cdot(-1)^{u+v} .
\end{aligned}
$$

Proposition 9. If $f \in A\left(U^{2}\right)$, then the error in the bivariate midpoint rule is

$$
\mathfrak{M}_{m, n}(f)-\mathfrak{I}(f)=Q_{m, \infty}(f)+Q_{\infty, n}(f)+Q_{m, n}(f)
$$

Proof. From (3.1) we get

$$
\mathfrak{M}_{m, n}(f)=\left(f, e_{0,0}\right)+Q_{m, \infty}(f)+Q_{\infty, n}(f)+Q_{m, n}(f) .
$$

Since $\mathfrak{I}(f)=\left(f, e_{0,0}\right)$, Proposition 9 follows.

Proposition 10. If $f \in E^{a}\left(U^{2}\right)$ with $a>1$, then the error in the bivariate midpoint rule satisfies

$$
\mathfrak{M}_{m, n}(f)-\mathfrak{I}(f)=\mathscr{O}\left(m^{-a}+n^{-a}\right) \quad(m, n \rightarrow \infty) .
$$

Proof. Since $f \in E^{a}\left(U^{2}\right)$, we have

$$
\begin{gathered}
Q_{m, \infty}(f)=\mathscr{O}\left(m^{-a}\right), \quad Q_{\infty, n}(f)=\mathscr{O}\left(n^{-a}\right), \\
Q_{m, n}(f)=\mathscr{O}\left(m^{-a} \cdot n^{-a}\right) \quad(m, n \rightarrow \infty),
\end{gathered}
$$

and (3.3) follows from Proposition 9.

Proposition 11. If $f \in \mathscr{C}_{0}^{q-1, q-1}\left(U^{2}\right) \cap \mathscr{C}^{q+1, q+1}\left(U^{2}\right)$ with $q \in \mathbb{N}$, then the error in the bivariate midpoint rule satisfies

$$
\mathfrak{M}_{m, n}(f)-\mathfrak{I}(f)=\mathscr{O}\left(m^{-q-1}+n^{-q-1}\right) \quad(m, n \rightarrow \infty) .
$$

Proof. The proof of Proposition 11 is similar to that of Proposition 4.

\section{4. $r$ TH-ORDER BLENDING MIDPOINT RULES}

We introduce the $r$ th-order sum of bivariate midpoint rules

$$
\mathbf{T}_{r}^{2}(f)=\sum_{m=0}^{r-1} \mathfrak{M}_{2^{m}, 2^{r-1-m}}(f) \quad(r \in \mathbb{N}) .
$$

Then the rth-order blending midpoint rule $\mathfrak{M}_{r}^{2}(f)$ is

$$
\mathfrak{M}_{r}^{2}(f)=\mathbf{T}_{r}^{2}(f)-\mathbf{T}_{r-1}^{2}(f),
$$

where $r \in \mathbb{N}$ and $r>1$. The construction of the $r$ th-order blending midpoint rule is analogous to the construction of the $r$ th-order blending rectangular rule. While the latter may be interpreted as an interpolatory cubature formula based 
on Boolean periodic spline interpolation, no such interpolatory characterization holds for the $r$ th-order blending midpoint rule.

The cubature points of $\mathfrak{M}_{r}^{2}(f)$ are mainly determined by the points occurring in $\mathbf{T}_{r}^{2}(f)$ :

$$
\bigcup_{m=0}^{r-1}\left\{\left((2 j+1) \cdot 2^{-m-1},(2 k+1) \cdot 2^{-r+m}\right): 0 \leq j<2^{m}, 0 \leq k<2^{r-1-m}\right\}
$$

Their number is given by

$$
m_{r}=r \cdot 2^{r-1} \text {. }
$$

Next we will determine a remainder formula for the $r$ th-order blending midpoint rule.

Proposition 12. If $f \in A\left(U^{2}\right)$, then the error in the rth-order blending midpoint rule is

$$
\begin{aligned}
\mathfrak{M}_{r}^{2}(f)-\mathfrak{I}(f)= & Q_{2^{r-1}, \infty}(f)+Q_{\infty, 2^{r-1}}(f) \\
& +\sum_{m=0}^{r-1} Q_{2^{m}, 2^{r-1-m}}(f)-\sum_{m=0}^{r-2} Q_{2^{m}, 2^{r-2-m}}(f) .
\end{aligned}
$$

Proof. In view of relations (3.2), (3.4), (4.1), and (4.3), the proof of (4.5) is similar to that of $(2.5)$.

Proposition 13. If $f \in E^{a}\left(U^{2}\right)$ with $a>1$, then the error in the rth-order blending midpoint rule is

$$
\mathfrak{M}_{r}^{2}(f)-\mathfrak{I}(f)=\mathscr{O}\left(r \cdot\left(2^{r-1}\right)^{-a}\right) \quad(r \rightarrow \infty) .
$$

Proof. In view of relations (3.4) and (4.5), the proof of (4.6) is similar to that of (2.6).

Remark 2. Recall that the number of cubature points of the $r$ th-order blending midpoint rule $\mathfrak{M}_{r}^{2}(f)$ is mainly determined by $m_{r}=r \cdot 2^{r-1}$. It is easily seen that the error relation (4.6) of the $r$ th-order blending midpoint rule obtains the form

$$
\mathfrak{M}_{r}^{2}(f)-\mathfrak{I}(f)=\mathscr{O}\left(\log \left(m_{r}\right)^{a+1} \cdot\left(m_{r}\right)^{-a}\right) \quad(r \rightarrow \infty),
$$

where $f \in E^{a}\left(U^{2}\right)$ with $a>1$. Thus, the $r$ th-order blending midpoint rule is comparable with the bivariate number-theoretic "good lattice" rules (see Sloan [5]). Again, the attractive feature of the $r$ th-order blending midpoint rule is its easy computation based on relations (4.1) and (4.2).

Proposition 14. If $f \in \mathscr{C}_{0}^{q-1, q-1}\left(U^{2}\right) \cap \mathscr{C}^{q+1, q+1}\left(U^{2}\right)$ with $q \in \mathbb{N}$, then the error in the rth-order blending midpoint rule satisfies

$$
\mathfrak{M}_{r}^{2}(f)-\mathfrak{I}(f)=\mathscr{O}\left(r \cdot\left(2^{r-1}\right)^{-q-1}\right) \quad(r \rightarrow \infty) .
$$

Proof. The proof of Proposition 14 is similar to that of Proposition 7. 


\section{A NUMERICAL EXAMPLE}

We consider the double integral

$$
\mathfrak{I}(f)=\int_{0}^{1} \int_{0}^{1} f(x, y) d x d y
$$

with the function

$$
f(x, y)=\frac{x+y}{1+x \cdot y} \quad(x, y \in U) .
$$

The function $f$ is an element of the Korobov space $E^{1}\left(U^{2}\right)$. Following Hua and Wang [4, p. 122] we introduce the function

$$
g(x, y)=\frac{1}{4}(f(x, y)+f(x, 1-y)+f(1-x, y)+f(1-x, 1-y)) .
$$

It is easily seen that

$$
\mathfrak{I}(g)=\mathfrak{I}(f)=2 \cdot(\log (4)-1)
$$

and

$$
g \in \mathscr{C}_{0}^{0,0}\left(U^{2}\right) \cap \mathscr{C}^{2,2}\left(U^{2}\right) .
$$

It follows from relation (1.8) that Propositions 4 and 7 are applicable to $g$ with $q=1$. The errors and the number of cubature points for the blending rectangle rule and the ordinary rectangle rule are shown in Table 1.

TABLE 1

Errors in blending and ordinary rectangle rules

\begin{tabular}{r|r|r|r|r}
\hline$r$ & $(r+1) \cdot 2^{r}$ & $\mathfrak{I}_{r}^{2}(g)-\mathfrak{I}(g)$ & $2^{2 r}$ & $\mathfrak{I}_{2^{r}, 2^{r}}(g)-\mathfrak{I}(g)$ \\
\hline 1 & 4 & 0.01009 & 4 & 0.01009 \\
2 & 12 & 0.00365 & 16 & 0.00282 \\
3 & 32 & 0.00120 & 64 & 0.00072 \\
4 & 80 & 0.00037 & 256 & 0.00018 \\
5 & 192 & 0.00011 & 1024 & 0.00005 \\
6 & 448 & 0.00003 & 4096 & 0.00001 \\
\hline
\end{tabular}

Similarly, it follows from relation (1.8) that Propositions 11 and 14 are applicable to $g$ with $q=1$. Table 2 shows the errors and the number of cubature points for the blending midpoint rule and the ordinary midpoint rule. In Figure 1 we exhibit the distribution of cubature points in $r$ th-order sum of midpoint rules.

Remark 3. It follows from (2.3) and (4.3) that the cubature points of $\mathbf{T}_{r}^{2}(f)$ form a subset of the cubature points of $\mathbf{S}_{r}^{2}(f)$ which are not contained in the set of cubature points of $\mathbf{S}_{r-1}^{2}(f)$.

Remark 4. The Boolean methods for double integration can be extended to arbitrary dimensions by using the method of $d$-variate Boolean interpolation developed in [2]. This is the topic of a forthcoming paper. 
TABLE 2

Errors in blending and ordinary midpoint rules

\begin{tabular}{r|c|c|r|c}
\hline$r$ & $r \cdot 2^{r-1}$ & $\mathfrak{M}_{r}^{2}(g)-\mathfrak{I}(g)$ & $2^{2 r-2}$ & $\mathfrak{M}_{2^{r-1}, 2^{r-1}}(g)-\mathfrak{I}(g)$ \\
\hline 1 & 1 & -0.02741 & 1 & -0.02741 \\
2 & 4 & -0.00317 & 4 & -0.00611 \\
3 & 12 & 0.00028 & 16 & -0.00148 \\
4 & 32 & 0.00035 & 64 & -0.00037 \\
5 & 80 & 0.00016 & 256 & -0.00009 \\
6 & 192 & 0.00006 & 1024 & -0.00002 \\
\hline
\end{tabular}
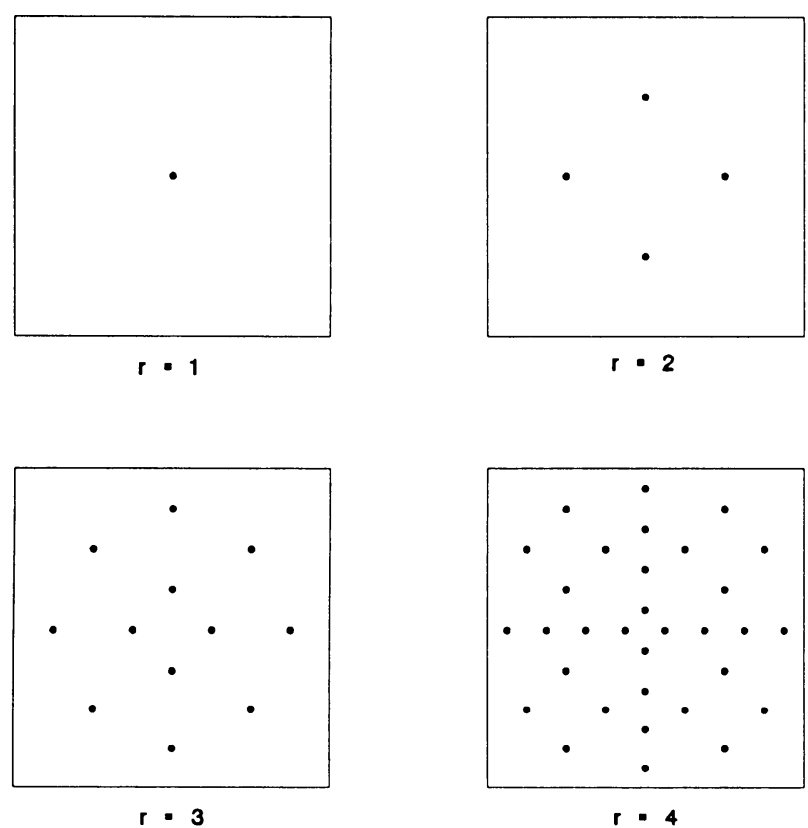

Figure 1

Points of rth-order sum of midpoint rules

\section{BIBLIOGRAPHY}

1. G. Baszenski and F.-J. Delvos, Boolean methods in Fourier approximation, Topics in Multivariate Approximation (C. K. Chui, L. L. Schumaker, and F. Utreras, eds.), Academic Press, 1987, pp. 1-11.

2. F.-J. Delvos, d-variate Boolean interpolation, J. Approx. Theory 34 (1982), 99-114.

3. F.-J. Delvos and H. Posdorf, $N$-th order blending, Constructive Theory of Functions of Several Variables (W. Schempp and K. Zeller, eds.), Lecture Notes in Math., vol. 571, Springer-Verlag, 1977, pp. 53-64. 
4. Hua Loo Keng and Wang Yuan, Applications of number theory to numerical analysis, Springer-Verlag, 1981.

5. I. H. Sloan, Lattice methods for multiple integration, J. Comput. Appl. Math. 12-13 (1985), 131-143.

Lehrstuhl für Mathematik I, Universität GH Siegen, Hölderlinstrasse 3, D-5900 Siegen, West Germany 